\title{
Indicadores dos serviços de abastecimento de água e esgotamento doméstico na Rota de Integração Latino-Americana
}

\author{
Indicators of water supply and sanitation services in the Latin American \\ integration Route
}

\section{Indicadores de los servicios de abastecimiento de agua y alcantarillado sanitario en la Ruta de Integración Latino-Americana}

\author{
Arlinda Cantero Dorsa ${ }^{1}$ \\ Mariana Antonio de Souza Pereira ${ }^{1}$ \\ Fernando Jorge Corrêa Magalhães Filho ${ }^{1}$
}

DOI: http://dx.doi.org/10.20435/inter.v21iespecial.2104

\begin{abstract}
Resumo: A análise de desempenho dos provedores públicos e privados de saneamento inseridos na rota de integração Latino-Americana, indica que as empresas privadas superam as públicas em investimentos e produtividade, contudo ambas necessitam de serviços especializados. As correlações existentes entre os indicadores indicam que quanto maior o atendimento do serviço, maior é a tarifa cobrada, e as perdas de água afetam diretamente no faturamento. Apesar dos avanços, ainda há necessidade de melhoria nos serviços. Palavras-chave: Corredor Bioceânico; desempenho; indicadores do saneamento; SNIS.

Abstract: The performance analysis of public and private sanitation providers included in the Latin American integration route indicates that private companies outperform public investments and productivity, both of which require specialized services. The correlations between the indicators indicate that the higher the service attendance, the higher the rate charged, and the water losses directly affect the billing. Despite progress, there is still a need for improvement in services.

Keywords: Bioceanic Highroad Crossing; performance; sanitation indicators; SNIS.

Resumen: El análisis de desempeño de los proveedores públicos y privados de saneamiento insertados en la ruta de integración latinoamericana, indica que las empresas privadas superan a las públicas en inversiones y productividad, sin embargo ambas necesitan servicios especializados. Las correlaciones existentes entre los indicadores indican que cuanto mayor sea la atención del servicio, mayor es la tarifa cobrada, y las pérdidas de agua afectan directamente en la facturación. A pesar de los avances, todavía hay necesidad de mejora en los servicios.
\end{abstract}

Palabras clave: Corredor Bioceânico; indicadores del saneamiento; rendimiento; SNIS.

\section{INTRODUÇÃO}

O acesso à água potável e ao esgotamento sanitário é um direito fundamental, mas as situações dos serviços relacionados ao saneamento básico ainda são consideradas precárias. Segundo o Serviço Nacional de Informações sobre Saneamento (SNIS), os dados mais recentes sobre a situação atual do saneamento no país indicam que mais da metade da população brasileira ainda não possui coleta de esgoto e somente $42,9 \%$ desse esgoto coletado é tratado. Quanto ao serviço de abastecimento de água apenas 83,3\% da população é atendida e o índice de perdas na distribuição é de 36,7\% (SNIS, 2016). Na área rural, em comunidades tradicionais os indicadores revelam uma situação ainda pior (MAGALHÃES FILHO; PAULO, 2017). Um dos setores que também é considerado deficiente é o transporte, que, incorporado à expansão das divisas produtivas, participa do elevado custo final dos produtos, principalmente relacionados a esfera agrícola e quando não há outras alternativas de modais. Então a busca de novas rotas

\footnotetext{
${ }^{1}$ Universidade Católica Dom Bosco, Campo Grande, Mato Grosso do Sul, Brasil.
} 
de escoamento dos produtos, que possibilitem a diminuição dos custos no mercado externo, se tornou uma realidade. Com isso a ideia dos corredores bioceânicos surgiu com intuito de integrar modais.

Os estudos sobre rotas de modo geral têm como objetivo encontrar a melhor forma de operação, importação, exportação de produtos e analisar os impactos sociais, econômicos e ambientais gerados. Montilha (2006) buscou alternativas para redução de custos por meio de novas rotas para melhoria da exportação e importação entre a Ásia e países Sul-Americanos. Oliveira (2011) analisou a perspectiva de construção da conexão ferroviária bioceânica entre portos e os impactos econômicos e sociais de investimentos em transportes, embora, não associado aos efeitos nas questões relacionadas ao abastecimento de água e esgotamento doméstico.

No âmbito do saneamento, encontram-se diversos estudos utilizando indicadores, no entanto voltados apenas para o serviço de abastecimento de água. Quando se trata do esgotamento sanitário os estudos possuem um foco em alternativas de tratamento. Em um estudo sobre indicadores do saneamento voltados ao serviço de abastecimento de água, sem incluir o esgotamento doméstico, RASERA et al. (2017) concluíram que os indicadores podem ser utilizados para exposição da qualidade dos serviços prestados pelas operadoras e como instrumentos robustos de melhoria para o planejamento e gestão.

Partindo do princípio de que a gestão dos serviços de saneamento deve ser avaliada, visando consolidá-la como um direito social dos cidadãos, busca-se, neste trabalho, avaliar o desempenho dos provedores públicos e privados, entre rotas que ligam dois municípios localizados na Região Centro-Oeste do Brasil, com base nos indicadores relacionados aos serviços de abastecimento de água e integrando com esgotamento sanitário. Seguindo três etapas: (i) correlação dos indicadores, (ii) análise da natureza jurídica e (iii) diagnóstico, a fim de obter informações úteis para tomada de decisão em projetos estratégicos de infraestrutura.

\section{METODOLOGIA}

O presente estudo buscou avaliar o desempenho dos provedores públicos e privados, no âmbito do saneamento, em duas rotas que ligam os municípios de Porto Murtinho a Campo Grande, localizados na Região Centro-Oeste do Brasil, uma pelo Corredor Bioceânico (BR-060 e MS-382) e a outra pela BR-262 e MS-339. Os municípios que compõem a Rota de Integração Latino-Americana, denominada também como Corredor Bioceânico (rota 1) são Jardim, Guia Lopes da Laguna, Nioaque, Maracaju, Anastácio, Dois Irmãos do Buriti e Sidrolândia. A rota 2, sendo uma outra opção de acesso, é composta pelos municípios de Bodoquena, Miranda, Aquidauana, Terenos, Anastácio e Dois Irmãos do Buriti (Figura 1). 
Figura 1 - Rotas de integração entre os municípios de Porto Murtinho à Campo Grande, Brasil

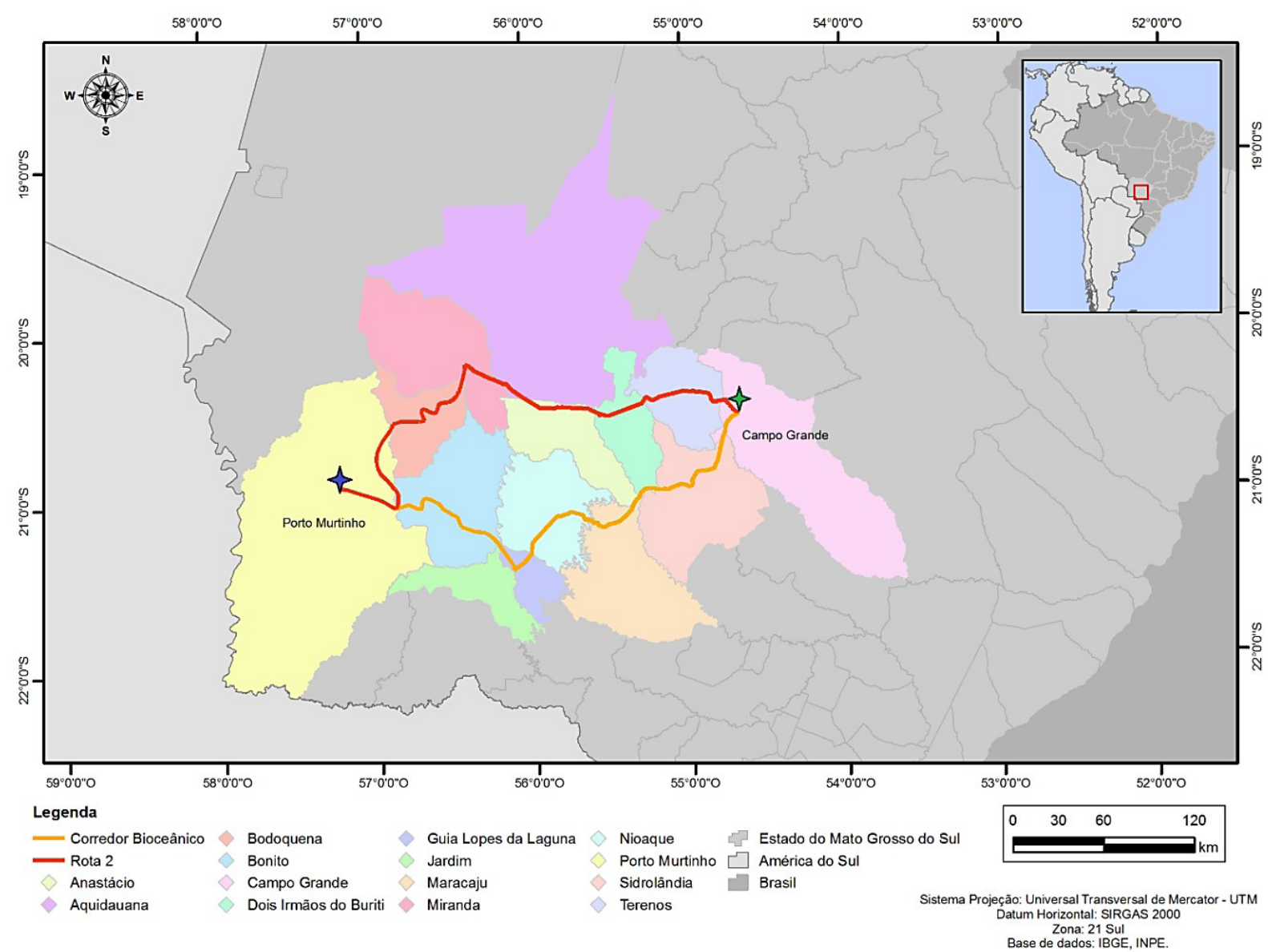

Fonte: IBGE, (s.d.).

Os indicadores de saneamento utilizados foram obtidos a partir de informações de domínio público disponibilizadas pelo banco de dados do Sistema Nacional de Informações sobre Saneamento (SNIS), no qual essas informações são organizadas a partir de declaração anual de responsabilidade dos próprios provedores do serviço. Os indicadores selecionados são apresentados na Tabela 1. Compreenderam o período de 2009 a 2016, levando em consideração a sua disponibilidade e como também utilizados em estudos na área (Pertel et al. (2016), Scriptore e Toneto (2012), Rasera et al. (2017) e Costa et al. (2013).

Tabela 1 - Indicadores e informações sobre abastecimento de água e esgotamento doméstico.

\begin{tabular}{clc}
\hline NÍVEL & \multicolumn{1}{c}{ INDICADORES } & UNIDADE \\
\hline & IN002- Índice de produtividade: economias ativas por & (econ./empreg.) \\
& pessoal próprio & $\left(\mathrm{R} \$ / \mathrm{m}^{3}\right)$ \\
& IN003- Despesa total com os serviços por m3 faturado & $\left(\mathrm{R} \$ \mathrm{~m}^{3}\right)$ \\
& IN005- Tarifa média de água & $\left(\mathrm{R} \$ \mathrm{~m}^{3}\right)$ \\
Econômico- & IN006- Tarifa média de esgoto & (percentual) \\
financeiro e & IN012- Indicador de desempenho financeiro & (econ./empreg. eqv.) \\
administrativo & IN019- Índice de produtividade: economias ativas por & $\left(\mathrm{R} \$ / \mathrm{m}^{3}\right)$ \\
& pessoal total (equivalente) & (percentual) \\
& IN026- Despesa de exploração por m3 faturado & (percentual) \\
& IN029- Índice de evasão de receitas & (ligações/empregados) \\
& IN101- Índice de suficiência de caixa &
\end{tabular}




\begin{tabular}{|c|c|c|}
\hline NÍVEL & INDICADORES & UNIDADE \\
\hline \multirow{7}{*}{$\begin{array}{l}\text { Operacionais de } \\
\text { água }\end{array}$} & IN009- Índice de hidrometração & (percentual) \\
\hline & IN013- Índice de perdas faturamento & (percentual) \\
\hline & IN023- Índice de atendimento urbano de água & (percentual) \\
\hline & IN028- Índice de faturamento de água & (percentual) \\
\hline & IN049- Índice de perdas na distribuição & (percentual) \\
\hline & IN055- Índice de atendimento total de água & (percentual) \\
\hline & $\begin{array}{l}\text { IN058- Índice de consumo de energia elétrica em sistemas } \\
\text { de abastecimento de água (SAA) }\end{array}$ & $\left(\mathrm{kWh} / \mathrm{m}^{3}\right)$ \\
\hline \multirow{4}{*}{$\begin{array}{l}\text { Operacionais de } \\
\text { esgoto }\end{array}$} & IN015- Índice de coleta de esgoto & (percentual) \\
\hline & IN016- Índice de tratamento de esgoto & (percentual) \\
\hline & IN046- Índice de esgoto tratado referido à água consumida & (percentual) \\
\hline & $\begin{array}{l}\text { IN059- Índice de consumo de energia elétrica em sistemas } \\
\text { de esgotamento sanitário (SES) }\end{array}$ & $\left(\mathrm{kWh} / \mathrm{m}^{3}\right)$ \\
\hline \multirow{2}{*}{ Qualidade } & $\begin{array}{l}\text { IN084- Incidência das análises de coliformes totais fora do } \\
\text { padrão }\end{array}$ & (percentual) \\
\hline & IN075- Incidência das análises de cloro residual fora do padrão & (percentual) \\
\hline \multirow{5}{*}{ Socioeconômico } & $\mathrm{PIB}$ & $(R \$ 1.000)$ \\
\hline & PIB per capita & $(R \$)$ \\
\hline & IDH-M & - \\
\hline & Índice Gini & - \\
\hline & INFORMAÇÕES FINANCEIRAS & UNIDADE \\
\hline \multicolumn{2}{|c|}{ FN014- Despesa com serviços de terceiros } & (R\$/ano) \\
\hline \multicolumn{2}{|c|}{ FN010- Despesa com pessoal próprio } & (R\$/ano) \\
\hline
\end{tabular}

Fonte: SNIS, 2016.

Para cada rota foi realizada uma matriz de correlação com os dados dos anos de 2009, 2010 e 2015, a fim de verificar o comportamento entre os indicadores selecionados, se há uma distinção ou se possuem as mesmas relações e, com isso estabelecer os indicadores mais influentes relacionados aos serviços de água e esgoto.

$$
R^{2}=\frac{\sum\left(\overleftrightarrow{Y}_{i_{-}} \bar{Y}\right)^{2}}{\sum\left(Y_{i_{-}} \overline{\bar{Y}}\right)^{2}}
$$

O coeficiente de associação $\left(R^{2}\right)$ estabelece uma relação entre os dados e modelo de reta que varia de 0 a 1. Quanto mais próximo de 1 mais forte é relação entre os dados, determinado pela equação 1 .

O algoritmo de aprendizado não supervisionado que agrupa dados com base em sua similaridade é o K Means Clustering. Busca encontrar padrões nos dados, no entanto se faz necessário especificar número de clusters que se deseja agrupar. O algoritmo atribui aleatoriamente cada observação a um cluster e localiza o centróide de cada cluster, através de duas etapas: (i) atribuir pontos de dados ao cluster cujo centróide está mais próximo e (ii) calcular o novo centróide de cada cluster. Esses dois passos são repetidos até que a variação dentro do cluster não possa ser mais reduzida. A variação dentro do cluster é calculada com a soma da distância euclidiana entre os pontos de dados e seus respectivos centróides de cluster. Conforme as expressões a seguir: 


$$
\mathrm{D}(\mathrm{Zp}, \mathrm{Mj})=\sqrt{\sum Z p, K y-M j, K y}
$$

Zp é ponto dos dados, MJ é centróide do cluster e Ky é o parâmetro de entrada.

$$
\mathrm{Mj}=\frac{1}{\mathrm{Nj} \sum Z p}, \nabla Z p \in C j
$$

Nj é o número de pontos de dados no cluster j.

A partir dos resultados da correlação os indicadores que apresentaram um coeficiente de correlação positiva acima de 0,5 foram selecionados. Foi então estabelecido 3 clusters para o agrupamento dados, pois as características jurídicas presentes nos dados são empresa privada (EP), organização social (OS) e sociedade de economia mista com administração pública (Semap). A partir dessa análise buscou-se avaliar a gestão do saneamento entre os provedores públicos e privados, comparando os seus desempenhos.

Uma das possíveis utilização dos indicadores são as medi-das quantitativas ou qualitativas, que podem ser empregadas na comparação da evolução histórica da qualidade do serviço ou para confrontarem os padrões atuais dos provedores, para o seu monitoramento (ALEGRE et al., 2004). Sendo assim, foi realizado um diagnóstico ao longo do tempo, com indicadores econômicos, operacionais e informações financeiras, os quais compreenderam os anos de 2009 a 2016.

\section{RESULTADOS E DISCUSSÃO}

\subsection{Correlação dos indicadores para tomada de decisão}

A partir da correlação (Figura 2), entre os indicadores considerando as correlações significativas tanto positivas como negativas, ou seja, aquelas que ocorrem no intervalo de 0,5-1, é possível verificar que a produtividade (INO2) se relaciona com a coleta e tratamento de esgoto (IN15, IN16 e IN46), despesa de exploração (IN26) como também com as demais produtividades (IN19, IN102), em ambas as rotas. Quanto às despesas com o serviço faturado (IN03), estas se associam com as despesas de exploração e indicador de desempenho financeiro (IN12), na rota 1 (Figura 2a). Na rota 2 (Figura 2b), além das despesas com exploração e desempenho financeiro, esse serviço se relaciona com a tarifa média de água.

A tarifa média de água (IN05) possui uma conexão com o PIB per capita (rota1), na rota 2 além desse indicador socioeconômico a despesa com a exploração também se associa a tarifa. Em ambas as rotas a tarifa se relaciona com o IG, IDHM e atendimento de água (IN23). Na rota 2 (Figura 2b) a tarifa média de esgoto (IN06) sofre influência dos indicadores operacionais de esgoto (IN15, IN16, IN46), na rota 1 (Figura 2a) esses mesmos indicadores operacionais juntamente com o índice de consumo de energia elétrica em sistemas de esgotamento sanitário (SES) e o $\mathrm{PIB}$, contribuem para o aumento dessa tarifa. Na rota 1 (Figura 2a) considerando o desempenho financeiro (IN12), esse indicador se relaciona com as perdas (IN13 e IN49) e faturamento de água (IN28) como também com a evasão de receitas (IN29). Na rota 2 (Figura 2b) somente o IN29 se relaciona ao desempenho financeiro. Observa-se que a única relação entre 0,5-1 na qualidade dos serviços, ocorre entre o IN75 e IN59, apontando que a incidência de cloro fora do padrão está ligado ao consumo de energia em sistemas de abastecimento de água (SAA) (Figura 2b). 
Figura 2 - Matriz de correlação

a)

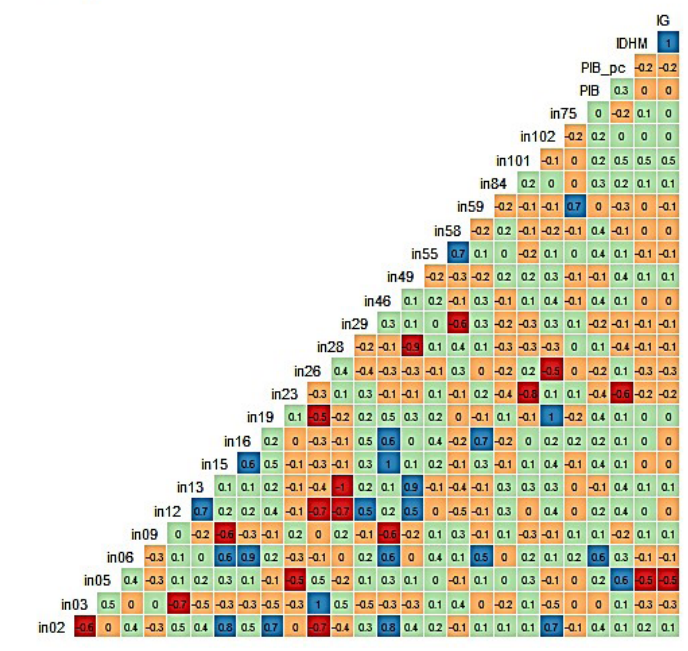

b)

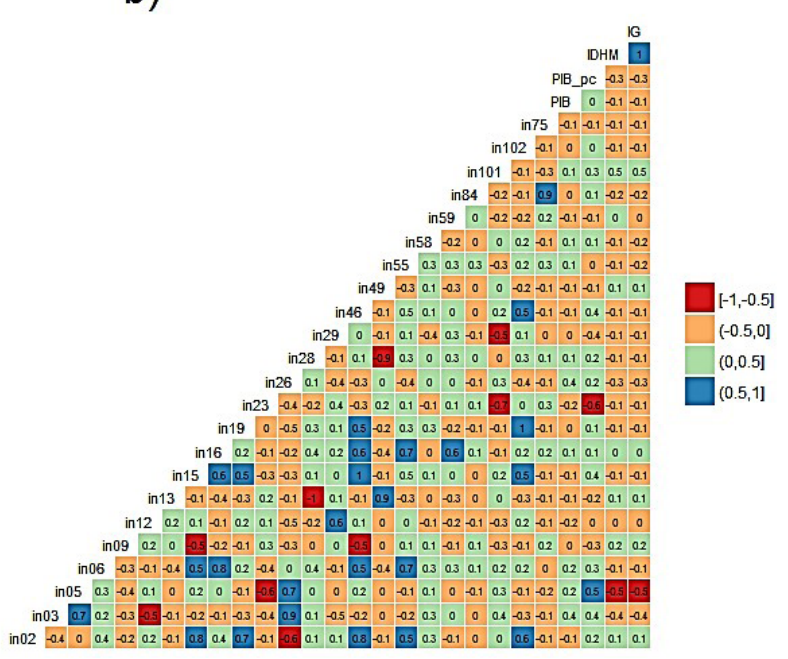

Fonte: SNIS, 2016.

Na rota 1 (Figura 2a), o índice disponível de atendimento de água (IN55) é influenciado pelo consumo de energia elétrica em SAA (IN58). Na rota 1 e na rota 2, as perdas (IN13 e IN49) se relacionam com o índice de faturamento (IN28). Em ambas as rotas se verifica a relação entre o índice de hidrometração (IN09) e os índices de operacionais de esgoto (IN15 e IN46), como também o atendimento de água (IN23) com o PIB per capita e o índice de suficiência de caixa (IN101). Somente na rota 1 (Figura 2a), a despesa com exploração (IN26) possui uma conexão com os indicadores econômico-financeiro e administrativo (IN19, IN2 e IN102), bem como o índice de evasão de receitas (IN29) com o consumo de energia em SAA (IN58) e suficiência de caixa (IN101). Nota-se a relação entre o índice de perdas faturamento e índice de perdas na distribuição, apresentando uma correlação negativa, ou seja, as perdas afetam diretamente no faturamento da prestadora, diminuindo seu lucro. Pertel et al. (2016) estabelecendo parâmetros de avalição de desempenho operacional relacionado a perdas no serviço de água, observaram que as perdas na distribuição foram superiores ou próxima às perdas no faturamento.

Outra relação verificada foi entre a tarifa média de água e o índice de atendimento de água, nessa relação, quanto maior o atendimento do serviço, maior é a tarifa cobrada. Nozaki (2007), em uma análise das iniciativas pública e privados internacionais e nacionais no setor de saneamento básico, conclui que, tantos casos internacionais quanto nacionais, como até mesmo onde não há a participação de capital privado, o aumento tarifário foi um fator fundamental e necessário para viabilização dos investimentos no setor, e que o aumento da tarifa é um fator comum quando há participação privada entre os prestadores de serviço, em algumas regiões do Brasil. Também avaliando a correlação entre indicadores no serviço de abastecimento de água, Fonseca e Gabriel (2015) verificaram que os valores de tarifação podem influenciar os indicadores operacionais como perdas de água na distribuição, sendo as maiores perdas no sistema de distribuição do que os serviços que não tarifam ou tarifam valores simbólicos pelo acesso à água, que pode ser medido pelo índice de atendimento de água. 


\subsection{Avaliação da natureza jurídica na gestão do saneamento}

No agrupamento dos clusters, pode-se verificar em dois casos os grupos formados foram OS e SEMAP e o outro somente EP. Esse comportamento se repetiu em todos os agrupamentos de indicadores para rota 1 . Para a rota 2 , o comportamento que se repetiu em todos os agrupamentos dos indicadores foi dois grupos formados por todas classes e um formado apenas com OS e Semap. Os indicadores analisados pertencem aos anos de 2009, 2010 e 2015, e isso indica que para a rota 1 o conjunto de dados pertencentes a EP possui um comportamento com uma diferença expressiva comparada com a OS e Semap. Para a rota 2, os dados são referentes à OS e Semap. Em alguns casos observou-se que o seu comportamento apresentou certa similaridade em comparação com a EP. Esses agrupamentos podem ser justificados pelo fato de em alguns casos as correlações entre alguns índices foram similares em ambas as rotas, como também em outros casos ocorrendo somente em uma rota.

\subsubsection{Análise financeira}

No desempenho financeiro entre as rotas, pode ser observado que quanto menor a tarifa média de água, menor foi o indicador de desempenho financeiro. Tanto na rota 1 quanto na rota 2 (Figura 3a, Figura 3b), a maioria dos dados possui um indicador de desempenho financeiro abaixo de $140 \%$, e quanto menor a tarifa média de água menor é esse indicador, ou seja, esse indicador sofre a influência da taxa mínima adotada por muitos provedores.

Com relação à tarifa média de esgoto boa parte dos dados estão concentrados na faixa de 1 a 2,5 R\$.m-3 (Figura 3d, Figura 3e) e há casos onde esse valor é zero, indicando a falta do serviço de coleta e tratamento de esgoto, e isso ocorre nos municípios de Dois Irmãos do Buriti, Guia Lopes da Laguna, Sidrolândia e Terenos. Verificou-se também que a despesa total com os serviços possui uma relação direta com a despesas de exploração. Onde observa-se um crescimento linear de ambas (Figura 3c, Figura 3f). Nota-se que tarifa média de água está entre a faixa de 3 a 4 R\$.m-3 (Figura 3a, Figura 3b). No entanto, Fonseca e Gabriel (2015) em uma análise da influência da tarifação em seis indicadores operacionais e de qualidade dos serviços de abastecimento de água entre os 5.178 prestadores no Brasil, observou que mais da metade está entre 1 e 3 R\$.m-3. Tanto o atendimento de água como PIB per capita dos municípios estudados apresentam-se acima da média nacional. Sendo 99,3\% o índice de atendimento de água, acima dos $83,3 \%$ da média nacional. O PIB per capita os municípios possuem em média R\$16.701,88 e a média nacional é de $\mathrm{R} \$ \mathbf{8 . 6 4 9 , 9 5}$. Isso justifica o fato das tarifas médias dos municípios serem maiores do que a média brasileira, pois conforme a relação encontrada no estudo entre o PIB per capita e a tarifa média de água, demostrando que o PIB per capita contribui para o aumento dessa tarifa, ou seja, relaciona o poder aquisitivo com o consumo, o qual aqueles que possuem um maior poder acabam consumindo mais água. 
Figura 3 - Cluster da análise financeira

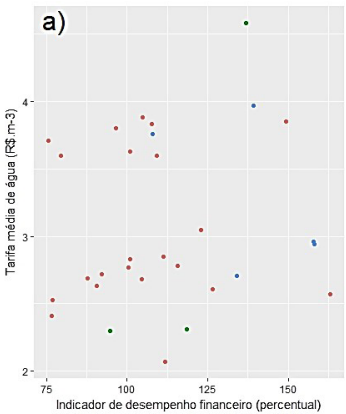

$$
\text { d) }
$$
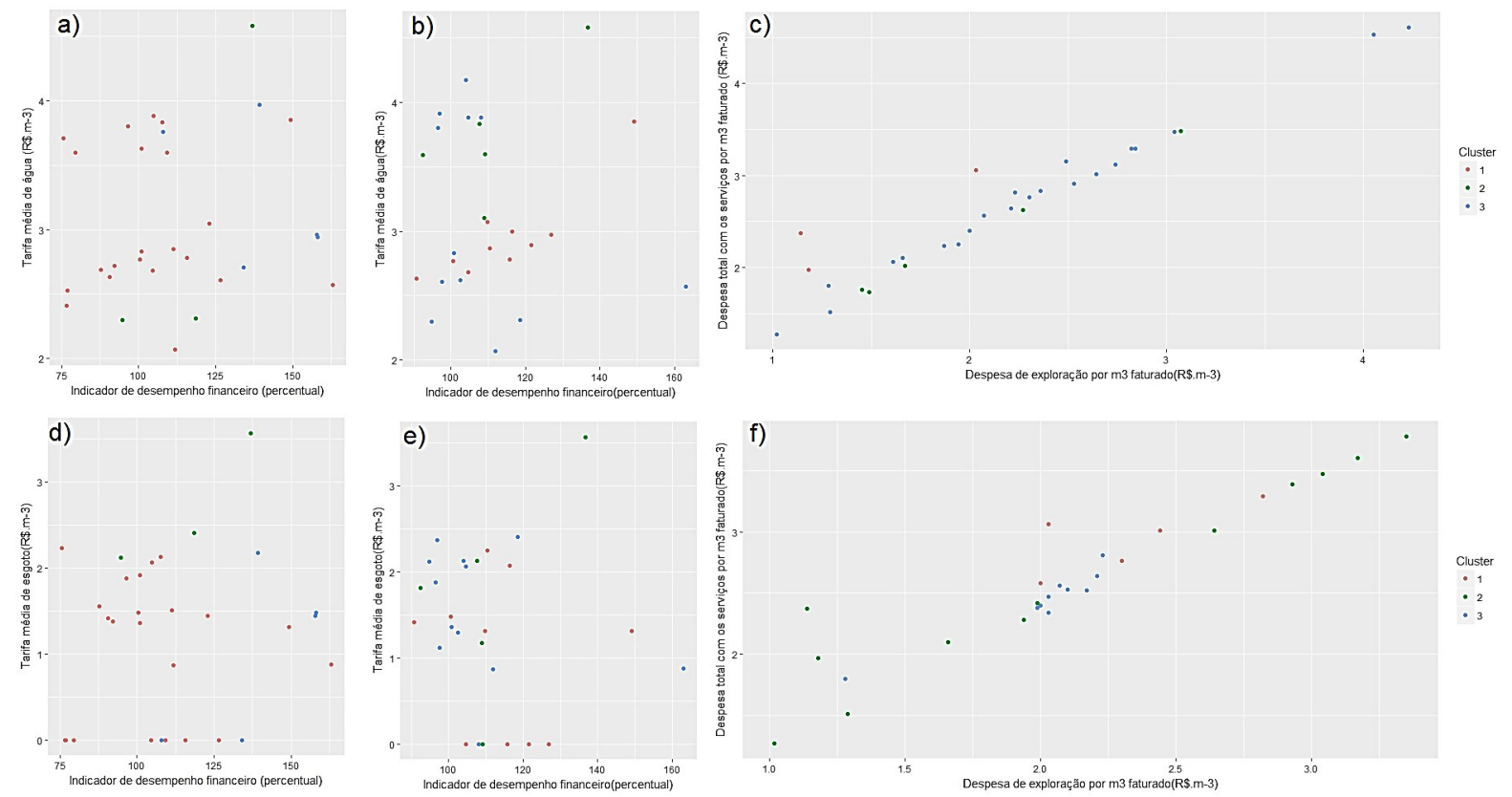

Fonte: SNIS, 2016.

\subsubsection{Análise operacional de água}

Nota-se que as perdas no faturamento possuem uma ligação direta com as perdas na distribuição (Figura 4c, Figura 4f). Comparando o comportamento operacional de água levando em consideração a distribuição e as perdas, observa-se que grande parte dos dados apresentam uma perda no faturamento e na distribuição de abaixo de 40\% (Figura 4a, Figura 4b, Figura 4d, Figura 4e). Para Tsutiya (2006), as perdas entre 25 e 40 \% são classificadas como regulares.

Figura 4 - Cluster da análise operacional de água

a)

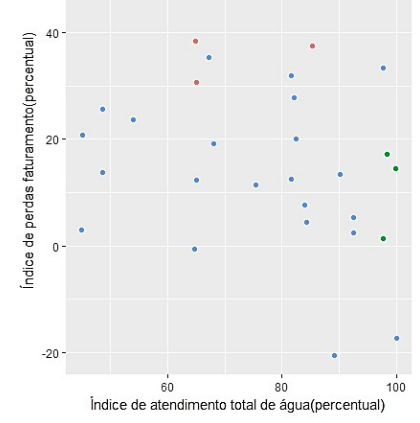

d)

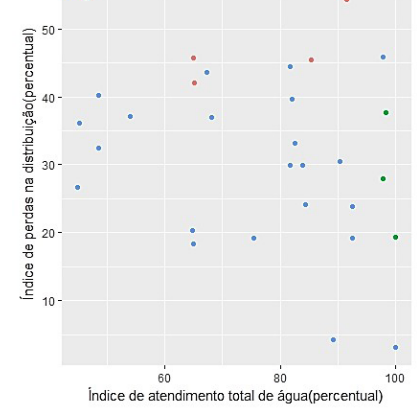

b)

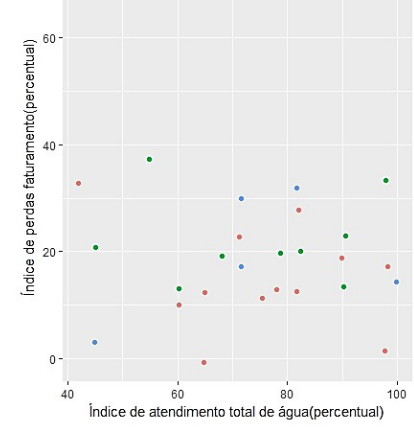

e)
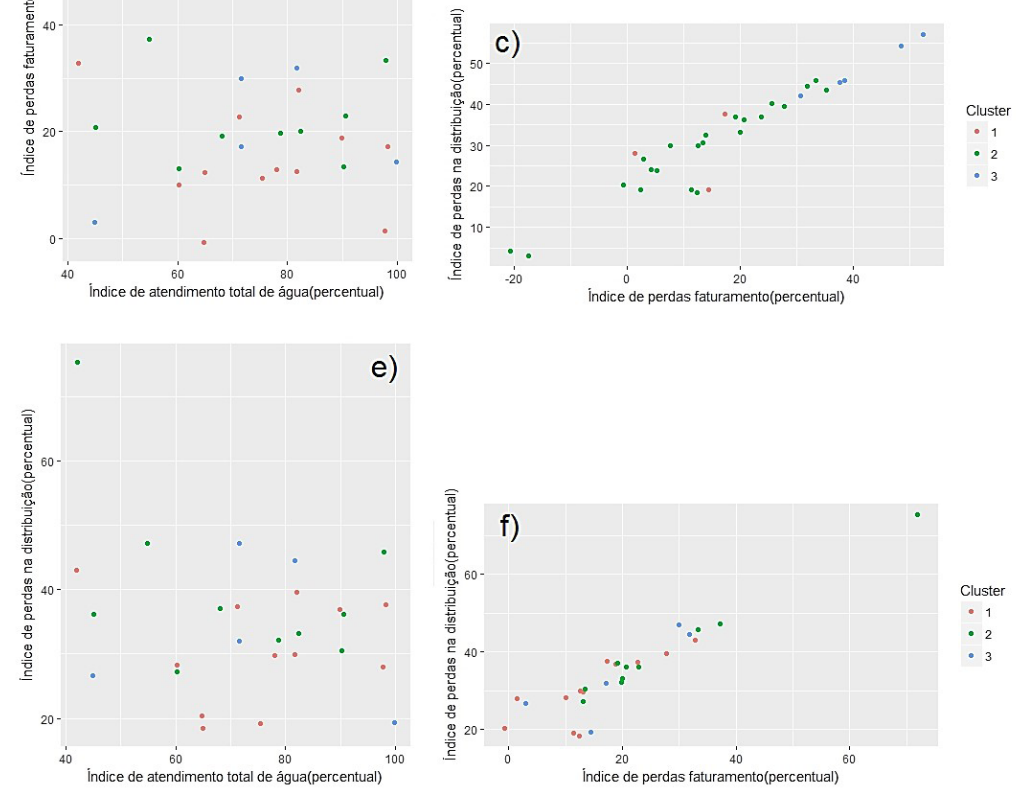

Fonte: SNIS, 2016. 
Kolbl et al. (2007) em um estudo sobre indicadores relacionados à perda de água em SAA, mostraram a importância do monitoramento de vazamento e vazamento ativo ao controle, pois as empresas que realizam mais esforços nessas tarefas obtêm melhores resultados de perdas do que outras. Baseado em um conjunto de dados de referência de diferentes sistemas distribuição de água em 20 países, Lambert e Mckenzie (2002) verificaram que fatores locais como localização de medidores, pressão média operacional e continuidade do fornecimento limitam o desempenho na gestão de perdas.

\subsubsection{Análise operacional de esgoto}

Com relação aos indicadores operacionais de esgoto, verificou-se que todo esgoto coletado é tratado (Figura 5a, Figura 5b). No entanto, observa-se que a rota 1 (Figura 2a), possui dois casos no cluster 3 e que esse tratamento ocorre abaixo de 100\%. Comparando o índice de consumo de energia no SES (Figura $5 c$, Figura $5 \mathrm{~d}$ ), grande parte dos dados se concentram entre 0,20 e 0,4 KWh.m-3.

Figura 5 - Cluster da análise operacional de esgoto
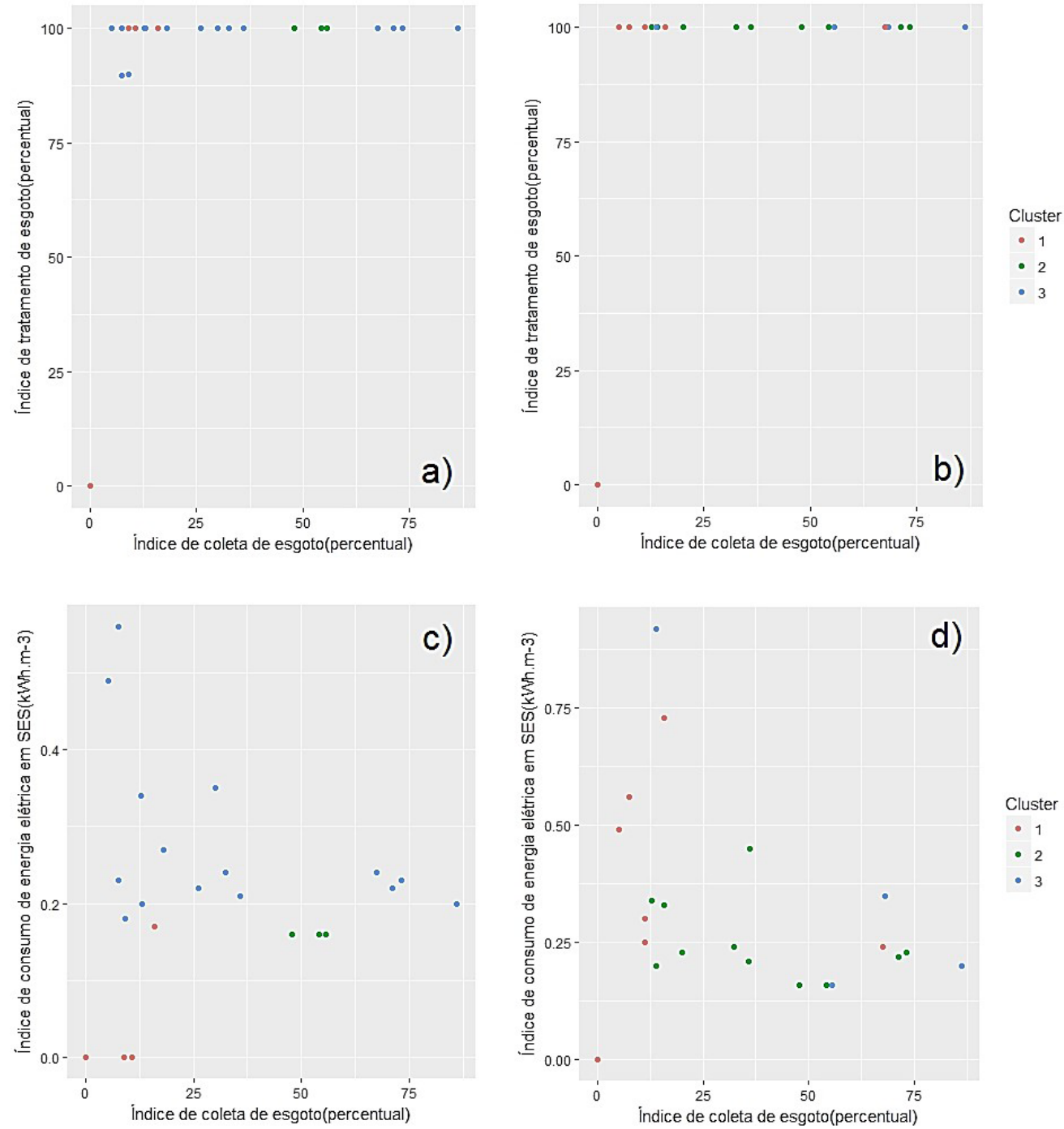

Fonte: SNIS, 2016 
Conforme Jordão (2014), nos sistemas de esgotamento sanitário, os custos de energia estão ligados às elevatórias e as estações de tratamento (ETEs). De acordo com os processos de tratamento, esse gasto com energia pode ser muito variado, em ETEs clássicas de lodos ativados, grande parte da energia utilizada está ligada ao processo de aeração. No Estado de Mato Grosso do Sul, o tratamento é realizado em grande parte por reatores UASB e lagoas de estabilização.

De acordo com referências internacionais, a parcela relacionada aos custos de energia nas Estações de Tratamento de Esgoto (ETEs) situa-se entre 25\% e 50\% dos custos operacionais (WEF, 2002).

\subsubsection{Análise da qualidade}

Quanto à qualidade do serviço de água, considerando a incidência de cloro residual fora do padrão, verificou que na maioria dos dados para rota 1 (Figura 6a) e rota 2 (Figura 6b), a incidência foi de zero. No entanto observa-se uma grande variação no atendimento total, mas o menor índice ocorre acima de 40\% (Figura 6a, Figura 6b), e isso deve-se ao fato de que parte dos municípios não possuírem atendimento de água nas áreas rurais. Farooq et al. (2008) buscaram determinar o relacionamento entre a presença de microrganismos e cloro residual na rede de distribuição. Verificou que a contagem microbiana foi inversamente proporcional ao cloro residual e com a concentração de cloro alta, a contagem microbiana diminuiu. Enquanto o aumento na turbidez resultou em aumento da contaminação microbiana. Em um estudo microbiológico para determinar a qualidade do abastecimento de água a uma comunidade urbana. De acordo com Agard et al. (2002), a existência de níveis médios de cloro residual no reservatório não garante o atendimento adequado às residências. Pois o nível de cloro residual diminuindo, do reservatório para rede doméstica, há um aumento significativo na prevalência de coliformes totais. São necessário estudos de modelagem hidráulica em redes de distribuição de água incluindo questões quali-quantitativas.

Figura 6 - Cluster da análise da qualidade

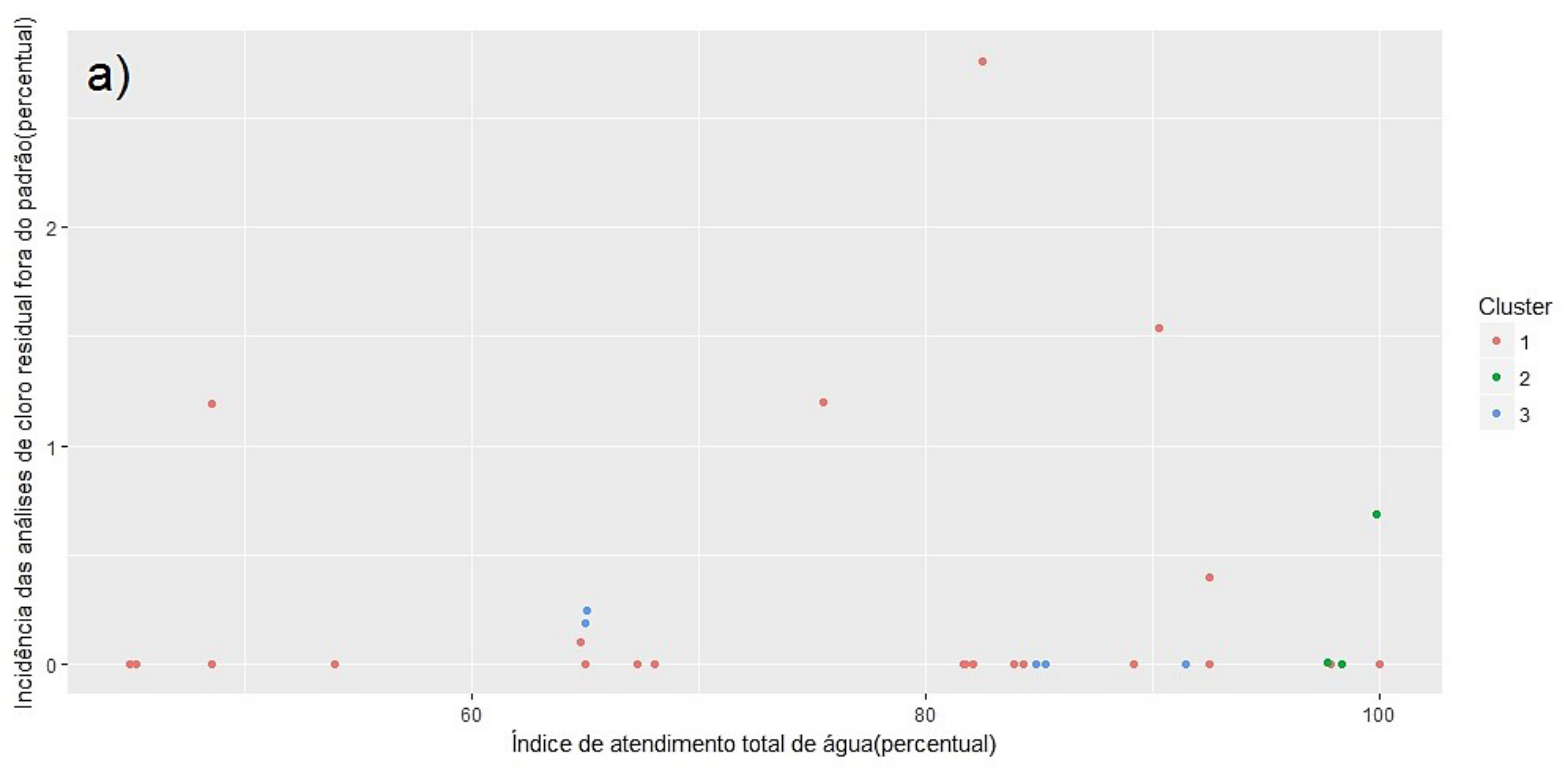




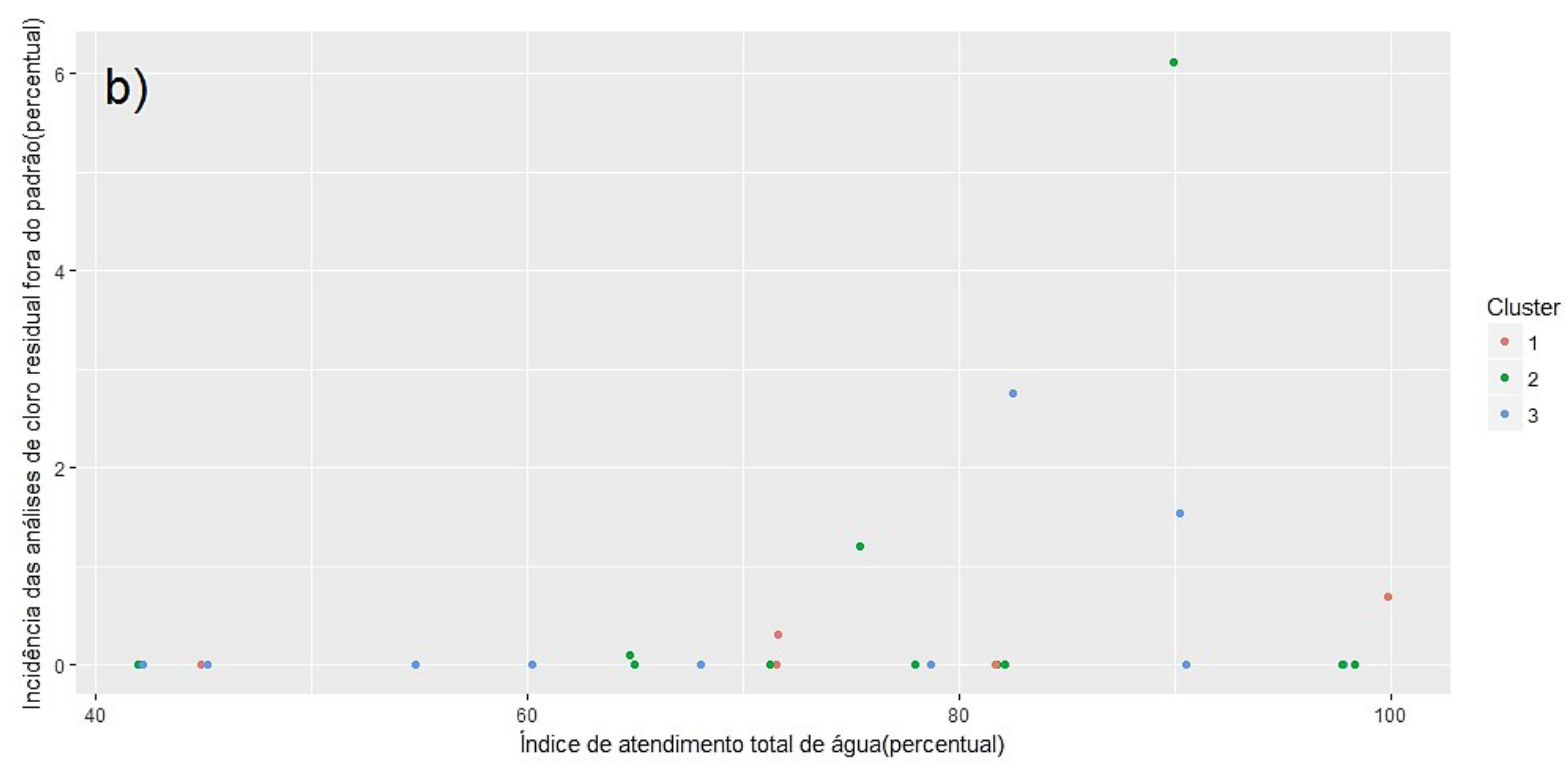

Fonte: SNIS, 2016.

\subsection{Diagnóstico ao longo do tempo}

No diagnóstico ao longo do tempo, relacionado aos indicadores econômicos, para a rota 1 (Figura 7) e rota 2 (Figura 8), pode ser verificado que o ano de 2008 apresenta uma maior presença de OS, já os demais anos a Semap obteve uma maior predominância, observando-se que os municípios que apresentavam OS passaram a ser providos por Semap. Ao se analisar o índice de produtividade total com o índice de produtividade do pessoal próprio (Figura 7a e 8a, Figura 7c e 8c) os municípios de Campo Grande e Bonito possuem uma maior produtividade de pessoal próprio. 
Figura 7 - Diagnóstico dos indicadores econômicos na rota 1
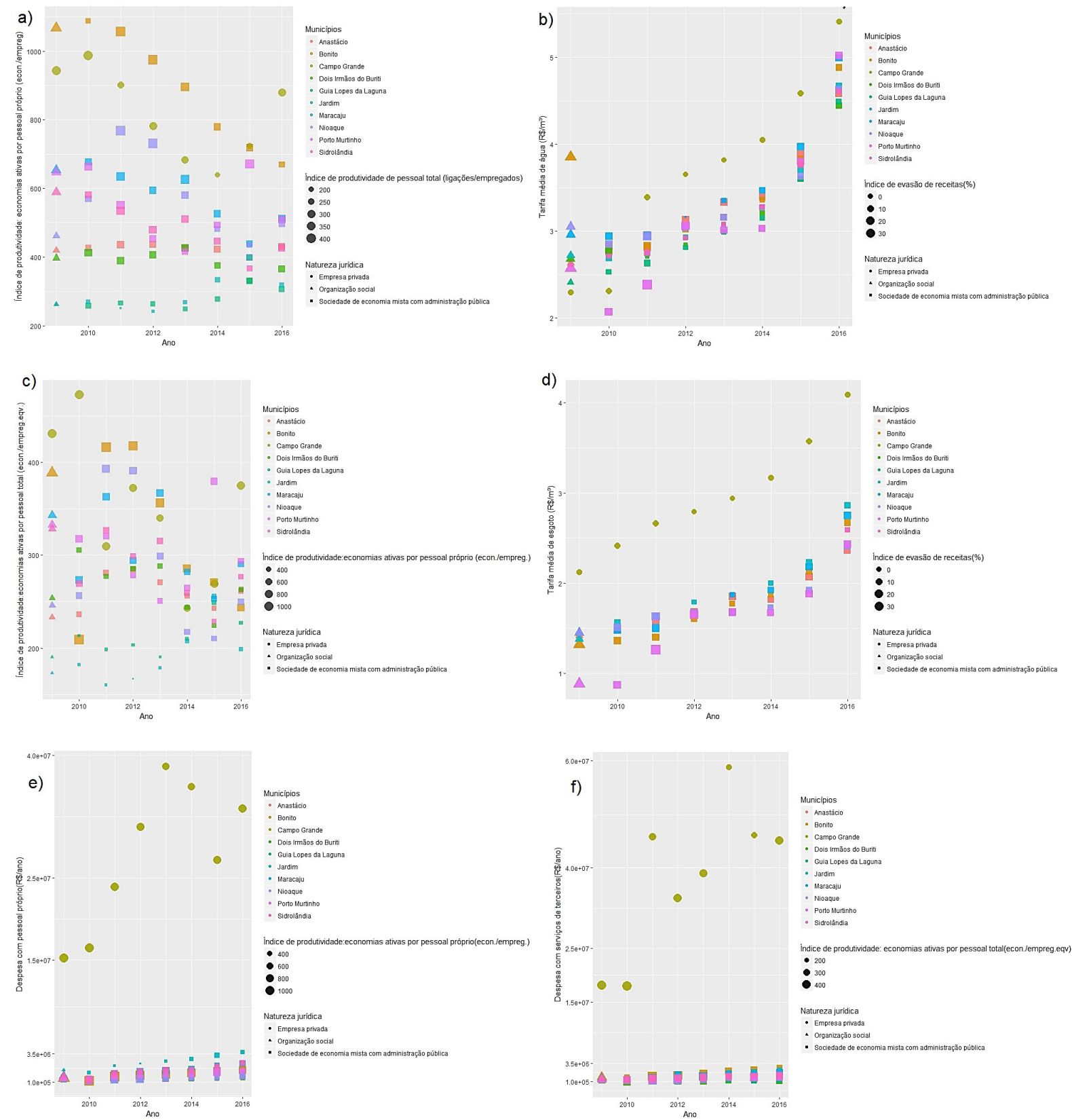

Fonte: SNIS, 2016.

Em âmbito jurídico considerando EP, nota-se que a despesa com o pessoal próprio e serviços de terceiros foram praticamente iguais para a os anos de 2009, 2010, 2012 e 2013. Nos anos de 2011, 2014, 2015 e 2016, as despesas com os serviços de terceiros são superiores do pessoal próprio. Para a OS e Semap, a despesa com o pessoal próprio e serviços de terceiros foram praticamente a mesma para todos os anos (Figura 7e e 8e, Figura $7 f$ e 8f). No caso da rota 1, percebe-se que no município de Jardim a despesa com o pessoal próprio evoluiu de forma linear (Figura 7e). Isso também ocorre na rota 2, no município de Aquidauana (Figura, 8e). Em relação às tarifas de água, esgoto e evasão de receitas (Figura 7b e 8b, Figura 7d e 8d) houve um aumento crescente nas tarifas, sendo as maiores tarifas da EP. As maiores evasões de receita são observadas na natureza jurídica OS e Semap. 
Figura 8 - Diagnóstico dos indicadores econômicos na rota 2
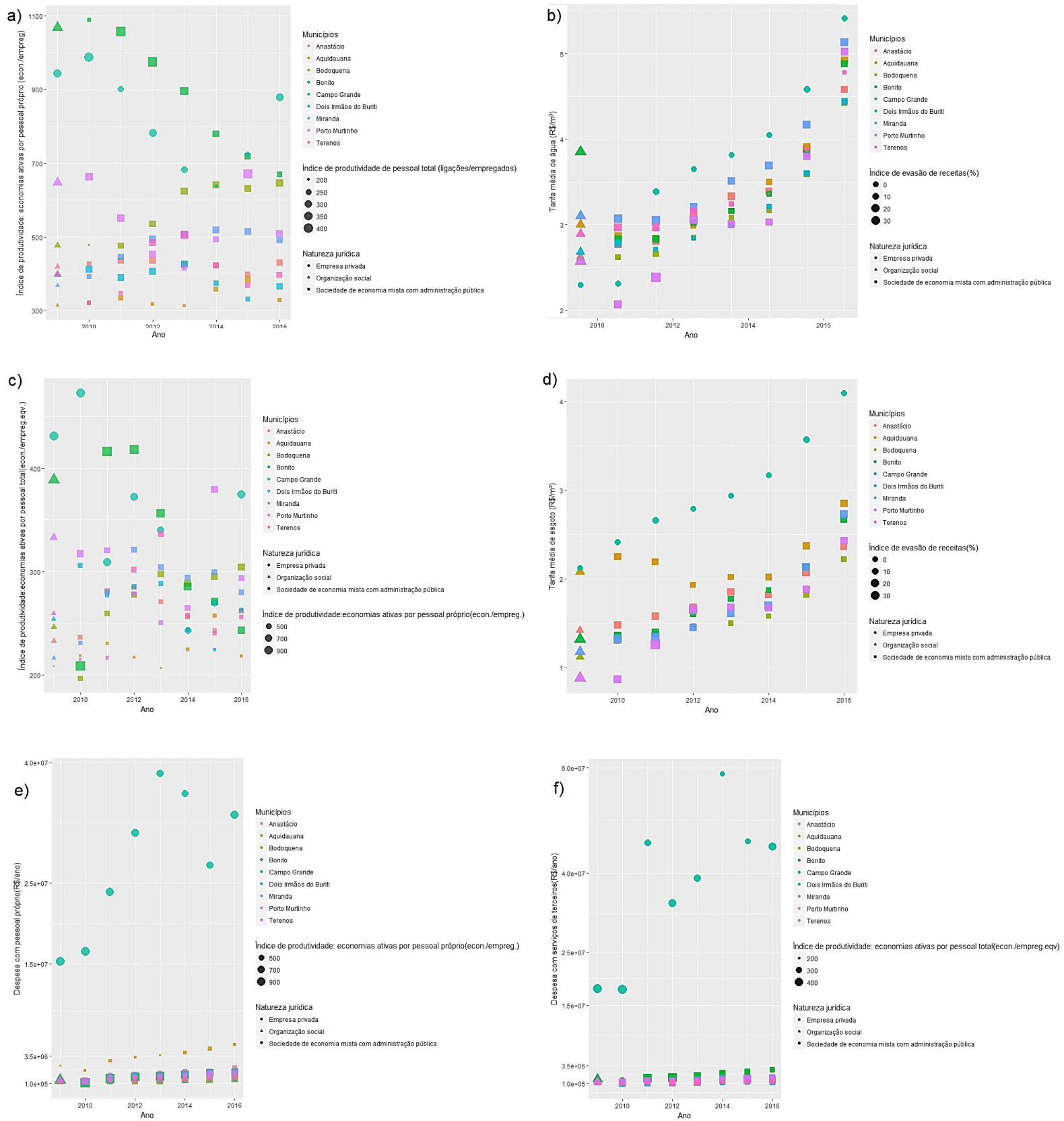

Fonte: SNIS, 2016.

Na rota 1, relacionando os indicadores operacionais de água como índice de faturamento e índice de hidrometração (Figura 9a), pode ser observado um aumento no faturamento a partir do ano de 2011 por parte da maioria dos municípios. O ano que apresentou o maior índice de faturamento foi de 2008, nos municípios de Guia Lopes da Laguna e Jardim. O menor foi em 2010 no município de Maracaju. Na rota 2 (Figura 9b), nota-se que o menor índice de faturamento de água foi registrado no ano de 2009, no município de Terenos, e o maior foi no município de Porto Murtinho, em 2014. Mas grande parte dos municípios apresentaram um índice de acima de 60\%. Os municípios que obtiveram os menores índices de hidrometração foram Bonito e Miranda, sendo 96 e 97\% respectivamente. 
Figura 9 - Diagnóstico dos indicadores operacionais de água
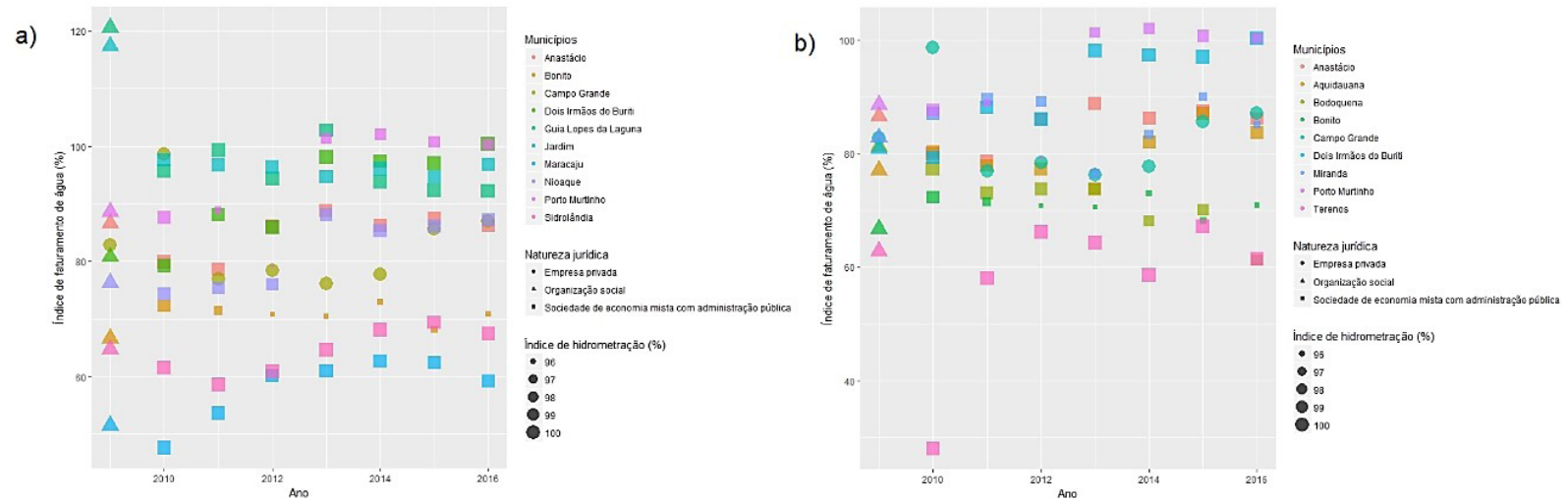

Fonte: SNIS, 2016.

A Figura 10a apresenta o diagnóstico ao longo do tempo relacionado aos indicadores operacionais de esgoto para a rota 1. Nota-se uma grande variação no índice de esgoto tratado referido a água consumida, mas de forma geral observa-se um aumento ao decorrer dos anos. Com relação ao índice de consumo de energia em SES, possuem valores entre 0,20 e 0,4 KWh.m-3. O maior consumo é verificado no município de Sidrolândia e o menor em Campo Grande e Maracaju.

Figura 10 - Diagnóstico dos indicadores operacionais de esgoto.
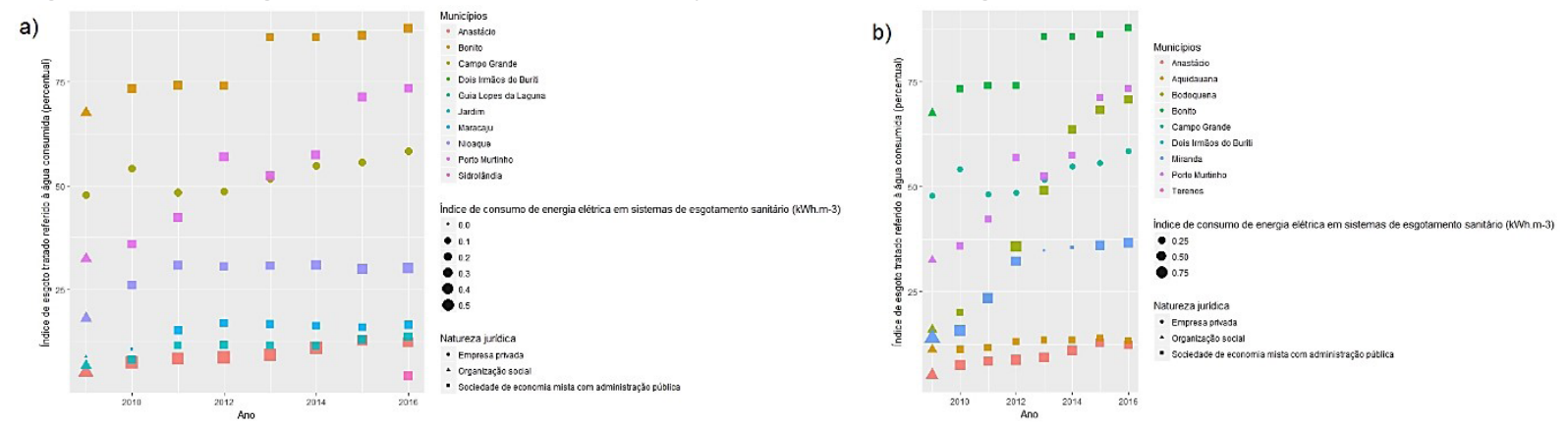

Fonte: SNIS, 2016.

Na rota 2 (Figura $10 b$ ) pode ser notado também um aumento no índice de esgoto tratado referido a água consumida, ao passar dos anos. Em relação ao índice de consumo de energia em SES, os valores estão entre 0,25 e 0,75 KWh.m-3, sendo a maior consumo nos municípios de Bodoquena, Miranda e Anastácio e o menor em Campo Grande e Miranda.

Analisando os determinantes da retração dos investimentos no setor de saneamento Toneto e Saiani (2006) observaram que os investimentos no saneamento básico, no Brasil, são influenciados por aspectos econômicos. Parte dos baixos índices de produtividade resultam da elevada utilização política dos provedores de serviços, o que também explica a maior tolerância com a inadimplência, a evasão de receitas e a prática de tarifas insuficientes para a cobertura de custos. Os mesmos autores observaram que os provedores regionais investiram mais que as públicas locais, pois possuíram melhores indicadores de tratamento de esgoto, no entanto suas tarifas médias são maiores. Os indicadores dos provedores privados apresentaram baixo atendimento, mas a capacidade de investimento e desempenho financeiro foi significativamente 
melhor do que os demais grupos. Por outro lado, os indicadores técnicos foram superiores, exceto os índices de produtividade de pessoal (TONETO; SAIANI, 2006).

Perard (2007), em estudo sobre a diferença de custo dos fundos, de transação da terceirização, diferença de eficiência e custo político potencial de privatização em 45 estados e 459 condados dos EUA, concluiu que operadores privados de água podem ser mais eficientes, em alguns casos, do que os governos locais por causa de uma diferença de conhecimento técnico e gerencial. A diferença de eficiência não é idêntica para todos os governos locais e depende da sua experiência. Essa diferença contribui para a escolha entre público e privado. Com uma abordagem diferente, baseada em indicadores estatísticos, Kirkpatrick et al. (2004) concluíram que a privatização pode levar a ganhos de desempenho, indicando que a propriedade privada pode implicar custos menores. Em uma análise comparativa da atuação de prestadores de serviços de saneamento públicos e privados do Brasil, Pinheiro et al. (2016) observaram também que os prestadores privados obtiveram melhor desempenho em uma série de aspectos como a produtividade, retorno e acesso aos serviços, e, com isso, concluiu que as concessões e parcerias público-privadas foram uma estratégia viável para alcançar a universalização do acesso aos serviços de saneamento.

\section{CONCLUSÃO}

O estudo buscou avaliar o desempenho dos provedores públicos e privados no âmbito do saneamento, por meio de indicadores e informações financeiras. Os resultados mostraram que mesmo o provedor público ou privado necessita de contratação de serviços especializados e com isso há uma diminuição da produtividade. Foi possível observar que a produtividade é maior na empresa privada quando comparado às públicas e isso está relacionado aos investimentos, pois as empresas privadas possuem uma maior autonomia, e os provedores públicos ficam dependentes dos repasses estatais. Com este investimento, o valor a ser pago pelo consumidor acaba sendo maior quando fornecido pela empresa privada, e elas por sua vez alcançam menores índices de evasão de receita.

Ao longo do tempo, no período estudado, os municípios que inicialmente apresentam organização social como natureza jurídica passaram a ser providos por sociedade de economia mista com administração e houve assim um aumento nas tarifas de água e de esgoto. A empresa privada difere da organização social e da sociedade de economia mista com administração em função das despesas com o pessoal próprio e serviços de terceiros. O índice de hidrometração nos municípios está entre 96-100\%, e de forma geral o faturamento de água apresenta-se acima de $60 \%$. Em âmbito operacional, evidências apontaram que grande parte das perdas no faturamento estão ligadas às perdas na distribuição e isso afeta o desempenho financeiro. Uma alternativa para o controle dessas perdas é o monitoramento e o controle dos vazamentos. Ocorreu um aumento no índice de esgoto tratado com o passar dos anos, e a média de consumo de energia no serviço entre os municípios é de 0,3 KWh.m-3. Para diminuição das tarifas relacionadas ao serviço de esgotamento sanitário, a construção de ETEs sustentáveis que geram um menor gasto de energia é uma opção. Em alguns municípios, a falta de alguns serviços como a coleta e tratamento de esgoto e o atendimento de água, ainda é uma realidade. 


\section{REFERÊNCIAS}

AGARD, L.; ALEXANDER, C.; GREEN, S.; JACKSON, M.; PATEL, S.; ADESIYUN, A. Microbial quality of water supply to an urban community in Trinidad. Journal of Food Protection, p. 1297-303, 2002.

ALEGRE, H.; HIRNER, W.; BAPTISTA, J. M.; PARENA, R. Indicadores de desempenho para serviços de abastecimento de água. Tradução de Patrícia Duarte, Helena Alegre e Jaime Melo Baptista. Lisboa: IWA Publishing, 2004. (Série de Guias Técnicos, 1).

COSTA, S. A. B.; CÔRTES, L. S.; COELHO, T.; FREITAS, M. M. Indicadores em saneamento: avaliação da prestação dos serviços de água e de esgoto em minas gerais. Revista UFMG, Belo Horizonte, v. 20, n. 2, p. 334-57, jul./dez. 2013.

FAROOQ, S.; HASHMI, I.; QAZI, I.A; QAISER, S.; RASHEED, S. Monitoring of coliforms and chlorine residual in water distribution network of Rawalpindi, Pakistan. Environ Monit Assess. p. 339-47, 2008.

FONSECA, A; GABRIEL, C. F. Análise da influência da tarifação em seis indicadores operacionais e de qualidade dos serviços de abastecimento de água no Brasil. Engenharia Sanitária e Ambiental, Rio de Janeiro, v. 20, n. 2, p. 219-24, 2015.

INSTITUTO BRASILEIRO DE GEOGRAFIA E ESTATÍSTICA (IBGE). Rio de Janeiro, [s.d.].

JORDÃO, E. P. Eficiência Energética em Tratamento de Esgotos. Revista DAE, São Paulo, p. 15-19, 2014.

KIRKPATRICK, C.; PARKER, D.; ZHANG, Y. F. State versus private sector provision of water services in Africa. World Bank Economic Review, v. 20, p. 143-63, 2004.

KOLBL, J.; THEURETZBACHER-FRITZ, H.; NEUNTEUFEL, R.; PERFLER, R.; GANGL, G.; KAINZ, H.; HABERL, R. Experiences with Water Loss Pis in the Austrian Benchmarking Project. Water Loss, p.176-87, 2007.

LAMBERT A. O; MCKENZIE R. D. Practical Experience in using the Infrastructure Leakage Index. Cyprus: Paper to IWA Managing Leakage Conference, 2002.

MAGALHÃES FILHO, F. J. C.; PAULO, P. L. Abastecimento de água, esgotamento doméstico e aspectos de saúde em comunidades Quilombolas no Estado de Mato Grosso do Sul. Interações, Campo Grande, MS, v. 18, n. 2, p. 103-16, 2017.

MONTILHA, A. C. Análise da influência logística na relação comercial entre Brasil e China: estudo de novas rotas para exportação pelo pacífico. Orientador: Sérgio Daroncho. 2006. Dissertação (Graduação em Logística) - Faculdade de Tecnologia da Zona Leste, São Paulo, SP, 2006.

NOZAKI, V. T. Análise do setor de saneamento básico no Brasil. Orientador: Rudinei Toneto Junior. 2007. Dissertação (Mestrado em Economia Aplicada)- Universidade de São Paulo, Ribeirão Preto, 2007.

OLIVEIRA, A. L. S. Saneamento básico no Brasil: limites e possibilidades de atuação do setor privado. Orientador: Antônio Herique Pinheiro Silveira. 2004. Dissertação (Mestrado em Economia)- Universidade Federal da Bahia, Salvador, BA, 2004.

OLIVEIRA, M. G. A Ferrovia Bioceânica Paranaguá-Antofagasta e a controvérsia entre o modelo primárioexportador e o modelo desenvolvimentista. OIKOS, Rio de Janeiro, v. 10, n. 2, 2011.

PERARD, E. Abastecimento de água: público ou privado? Uma abordagem baseada no custo dos fundos, custos de transação, eficiência e custos políticos. In: CONFERÊNCIA SOBRE O PAPEL DO ESTADO NA PRESTAÇÃO DE SERVIÇOS. Escola de Políticas Públicas Lee Kuan Yew, Cingapura. 2007. Anais [...]. Cingapura, 2007. 
PERTEL, M.; AZEVEDO, J. P. S. D.; VOLSCHAN JUNIOR, I. The use of water loss index as an indicator for benchmarking: the case of Brazilian water supply state companies. Engenharia Sanitaria e Ambiental, Rio de Janeiro, v. 21, n. 1, p. 159-68, 2016.

PINHEIRO, F. A. P.; SAVOIA, J. R. F.; DE ANGELO, C. F. Análise Comparativa da Atuação de Prestadores de Serviços de Saneamento Públicos e Privados no Brasil. Brazilian Business Review, v. 13, n. 1, p. 118-40, 2016.

RASERA, D; MENDES, T. G; CETRULO, T; YOSHII, M. P. C; MALHEIROS, T. F; MOREIRA, R. M; MENDIZÁBALCORTÉS, A. D; GUIMARÃES, E. F. Indicadores para Regulação do Saneamento em Áreas de Pobreza: Estrutura e Aplicação em Cubatão-SP, Brasil. Revista Ambiente \& Sociedade, São Paulo, v. 20, n. 4, 2017.

SCRIPTORE, J. S.; TONETO, R. J. A estrutura de provisão dos serviços de saneamento básico no Brasil: uma análise comparativa do desempenho dos provedores públicos e privados. Revista de Administração Pública, Rio de Janeioro, v. 46, n. 6, p. 1479-504, Rio de Janeiro, 2012.

SISTEMA NACIONAL DE INFORMAÇÕES SOBRE SANEAMENTO (SNIS). Diagnóstico dos serviços de água e esgotos (anos-base 2008 a 2016). Brasília: Ministério das Cidades, 2016.

TONETO, R. J; SAIANI, C .C. Restrições à expansão dos investimentos no saneamento básico brasileiro. Revista Econômica do Nordeste, Fortaleza, v. 37, n. 4, 2006.

TSUTIYA, M. T. Abastecimento de água. 4. ed. São Paulo: Departamento de Engenharia Hidráulica da Escola Politécnica da Universidade, p. 643, 2006.

WATER ENVIRONMENT FEDERATION (WEF). Activated Sludge. Manual of practice: operations and maintenance, n. 9. WEF, 2002.

\section{Sobre os autores:}

Arlinda Cantero Dorsa - Doutora em Língua Portuguesa pela Pontifícia Universidade Católica de São Paulo (PUC-SP). Professora na Universidade Católica Dom Bosco (UCDB). E-mail: acdorsa@ucdb.br, Orcid: http://orcid.org/0000-0002-1120-0273

Mariana Antonio de Souza Pereira - Graduada em Engenharia Sanitária e Ambiental pela Universidade Católica Dom Bosco (UCDB). E-mail: marianapereira.esa@gmail.com, Orcid: http://orcid.org/0000-0002-9415-5374

Fernando Jorge Corrêa Magalhães Filho - Doutor em Saneamento Ambiental e Recursos Hídricos pela Universidade Federal de Mato Grosso do Sul (UFMS). Professor na Universidade Católica Dom Bosco (UCDB). E-mail: fernando@ucdb.br, Orcid: http://orcid.org/0000-0001-5447-0137 
